\title{
ECONOMIA MATEMATICA E MECCANICA RAZIONALE
}

\author{
PIERCARLO NICOLA ${ }^{1}$
}

RiAssunto. - Almeno a partire da Pareto le analogie meccaniche hanno giocato un ruolo rilevante nella teoria economica; ma sarebbe ingiusto passare sotto silenzio il fatto che Marshall, praticamente coetaneo di Pareto, propendeva piuttosto per l'impiego di analogie di natura biologica. Questo detto, l'equilibrio generale, attualmente il paradigma più diffuso fra $\mathrm{i}$ cultori di teoria economica, si presta mirabilmente a sottolineare le analogie meccaniche proponibili nella teoria economica.

Partendo dalla formulazione canonica dell'equilibrio generale concorrenziale, come venne proposta da Arrow e Debreu nel 1954, l'intervento si propone di presentare succintamente alcune delle estensioni formulate nel corso degli ultimi 50 anni: - l'introduzione dei monopoli e della concorrenza monopolistica;

- i prezzi rigidi e il razionamento;

- le generazioni sovrapposte;

- gli equilibri temporanei e le aspettative soggettive;

- gli equilibri approssimati in presenza di non convessità;

- l'infinità degli agenti e la concorrenza perfetta;

- l'incertezza;

- i mercati incompleti e le attività finanziarie.

Vengono brevemente discussi anche i problemi che sorgono nell'applicazione di modelli di equilibrio generale allo studio di economie concrete, e si sottolinea il fatto che i dati economici sono sempre soggetti ad errori spesso rilevanti, mentre quasi mai é possibile condurre esperimenti controllabili, diversamente da quanto avviene nella fisica. Le applicazioni portano naturalmente a chiedersi quale utilità possono rivestire i modelli di equilibrio generale a fini previsionali.

ABSTRACT. - Since Pareto times mechanical analogies played a relevant role in economic theory; but it is necessary to underline that Marshall, an economist contemporary to

1 Ringrazio gli intervenuti all'Incontro di Studio per le osservazioni ricevute, che mi hanno consentito di meglio specificare e correggere alcuni punti della relazione. 
Pareto, preferred to employ biological analogies. General equilibrium, presently the mainstream paradigm in economic theory, lends itself very naturally to underline mechanical analogies permeating economic theory.

Starting from the canonical formulation of general equilibrium, as proposed by Arrow and Debreu in 1954, our aim is to briefly recall some extensions made during the last 50 years:

- the inclusion of monopolies and monopolistic competition;

- sticky prices and rationing;

- overlapping generations;

- temporary equilibrium and subjective expectations;

- approssimate equilibria under non convexities;

- infinity of agents and perfect competition;

- uncertainty;

- incomplete markets and financial activities.

We discuss also some problems which are present in applying general equilibrium models to our days economies, and we underline the fact that economic data are always affected by errors, while very rarely it is possible to do controlled experiments, contrary to what happens in physics. Therefore, it is natural to ask which is the utility of general equilibrium models as far as forecasting activities are of interest.

\section{FISICA E CHIMICA}

Quando l'economia politica, dopo avere compiuto i primi passi sostanziali con Adam Smith(1776), aspirò diventare scientifica, vide in Antoine Augustin Cournot(1838) il primo studioso veramente capace di rivestirla di un formalismo matematico adeguato ai tempi. Cournot era un professore universitario di meccanica, curioso più che esperto di temi economici, ma non vi é dubbio che la sua monografia segnò per gli economisti l'avvio del riconoscimento della fisica, in modo particolare della meccanica razionale, come paradigma di riferimento per le formalizzazioni della teoria economica.

Vilfredo Pareto (Parigi1848, Celigny1923), nato scientificamente come ingegnere con studi preliminari di matematica, fu assertore convinto dell'impiego di analogie meccaniche nelle sue ricerche sull'economia matematica, anche se non mancò di sottolineare che talvolta le analogie di natura biologica potevano essere promettenti. Nel pensiero di Pareto é fondamentale la contrapposizione fra gusti e ostacoli, con queste due entità idealmente collocate sui due piatti di una bilancia da porre in equilibrio attraverso opportuni accorgi- 
menti. I gusti, rappresentati dalle ofelimità ${ }^{2}$ degli agenti consumatori, generano le funzioni di domanda, mentre gli ostacoli, rappresentati dalle funzioni di produzione delle imprese, generano le funzioni di offerta.

Fu invece Alfred Marshall (Bermondsay 1842, Cambridge 1924) a fare sovente riferimento alla biologia (spesso da lui indicata come chimica) quale fonte di ispirazione per l'economista, anche se non mi sembra che egli se ne sia sistematicamente servito. La predilezione di Marshall per la biologia é ben documentata dal seguente passo:

La Mecca dell'economista sta piuttosto nella biologia economica che nella dinamica economica. Ma i concetti biologici sono più complessi di quelli della meccanica; un volume sui fondamenti deve dare quindi un posto relativamente largo alle analogie meccaniche; e sarà fatto uso frequente della parola "equilibrio", che suggerisce qualcosa di un'analogia statica. (Marshall, p.VII della Prefazione alla ottava edizione, tradotta in Italiano da Alberto Campolongo).

É indubbio che tanto in economia quanto in biologia si presentano frequentemente argomenti nei quali svolgono un ruolo importante problemi di massimizzazione vincolata. Ma questo riconoscimento non sembra possa bastare per assegnare un ruolo fondamentale ai principi della biologia nello studio di problemi economici, nonostante gli importanti contributi di Simon, per esempio Simon(1955). Vi sono delle perplessità verso le analogie di carattere biologico in economia, dovute al fatto che gli organismi viventi hanno tutti un ciclo preciso e ineluttabile: nascita, crescita, invecchiamento, estinzione. Invece i sistemi economici, una volta nati, si perpetuano, sia pure attraverso fasi di espansione e contrazione; ma ad ogni contrazione prima o poi segue nuovamente una fase di espansione. Nella storia umana moderna non sembra si siano presentati casi di economie che si sono estinte, almeno per cause naturali.

Si possono illustrare le differenze fra l'andamento temporalmente limitato della vita di un organismo vivente, e l'andamento tem-

2 Questo é il termine preferito da Pareto, mentre oggi si denominano funzioni di utilità. L'utilità é una grandezza che ordina, ma non misura, i possibili vettori che rappresentano le quantità consumate dei vari beni. Nonostante questo, dalla massimizzazione vincolata di una funzione di utilità si ottengono funzioni di domanda cardinali. 
poralmente esteso indefinitamente nel futuro di un'economia, attraverso le Figure 1 e 2 seguenti:

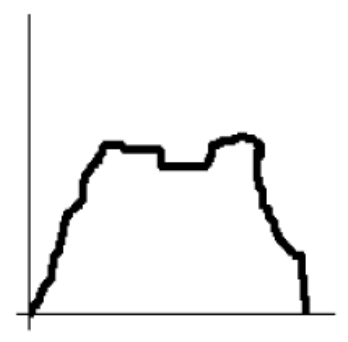

Figura 1. Individuo.

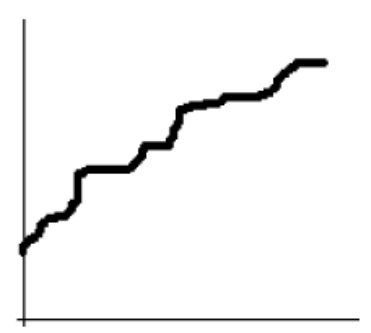

Figura 2. Economia.

In entrambe le Figure l'asse delle ascisse indica il tempo; nella Figura 1 l'asse delle ordinate rappresenta un "indice di vitalità", mentre nella Figura 2 sull'asse delle ordinate si può pensare sia rappresentato il cosidetto PIL, ossia il prodotto interno lordo.

Questo detto, si deve riconoscere che l'idea di organismo biologico e della sua evoluzione può ben applicarsi agli agenti economici singoli, le imprese e i consumatori. Per fare due esempi, la teoria del ciclo vitale del consumatore, proposta da Modigliani e Brumberg (1954), contiene in nuce aspetti che si possono riferire alle fasi della vita di molti organismi viventi, mentre ogni impresa sembra essere soggetta ad una inevitabile evoluzione temporale che manifesta esplicitamente le fasi nascita, crescita,declino, estinzione(per raggiunti obiettivi sociali nel migliore dei casi, oppure per fallimento). ${ }^{3}$

Si può ulteriormente illustrare la differenza di fondo fra Pareto e Marshall ricordando che Pareto richiama spesso il termine "efficienza", mentre il termine usato da Marshall é "benessere". Il termine efficienza ha una connotazione molto importante nella teoria economica odierna, ${ }^{4}$ ma é assai meno stringente del termine benessere, che in un certo modo sembra richiamare la misurabilità delle funzioni di utilità.

3 Su aspetti biologici della teoria dell'impresa si veda Penrose (1952).

4 L'efficienza paretiana di un equilibrio generale concorrenziale afferma che in equilibrio, date le risorse disponibili, non é possibile aumentare l'utilità di un consumatore se non diminuendo quella di almeno un altro consumatore. 


\section{TEORIA ECONOMICA E MECCANICA(RAZIONALE)}

Max Plank, premio Nobel per la fisica, dovendo scegliere la facoltà universitaria cui iscriversi, pare fosse indeciso tra fisica ed economia. Da buon Tedesco lesse alcuni libri di economia e altri di fisica; decise per la fisica, in quanto la ritenne disciplina meno difficile e complicata dell'economia. Questo piccolo aneddoto sottolinea abbondantemente il fatto che l'economia, essendo una scienza umana, é continuamente soggetta alle scelte, non sempre razionali, degli esseri umani, e quindi la struttura di ogni sistema economico si evolve continuamente nel tempo, a differenza di quanto accade nell'universo fisico, il quale ubbidisce a leggi che spesso sono indubbiamente estremamente complicate, ma che rimangono immutabili nel tempo e nello spazio, dunque con una struttura definitivamente fissata sin dall'origine dei tempi.

Un'idea della complicazione cui é sottoposta l'economia può risultare richiamando l'oggetto (fisico) bilancia. Una bilancia consta di due piatti, posti a uguale distanza dal fulcro sul quale é imperniato un braccio rigido, come in Figura 3. Due quantità di materia, $p$ e $q$, sono poste su ogni piatto e le dette quantità hanno lo stesso peso quando $i$ piatti sono perfettamente allineati, senza con questo conoscere quale sia il comune peso delle due sostanze (analisi qualitativa). Se però una delle due quantità é costituita da un peso di valore precisato, in equilibrio anche l'altra sostanza ha esattamente il peso della quantità di riferimento (analisi quantitativa).

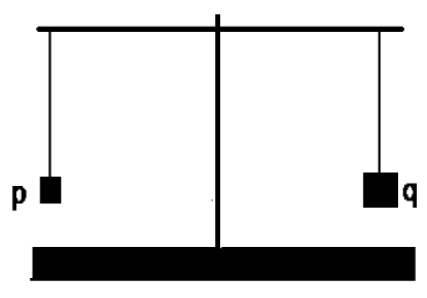

$\mathbf{p}=\mathbf{q}$

Figura 3. Bilancia meccanica.

In economia, se vogliamo considerare il funzionamento di un intero sistema economico, nel quale si producono, scambiano e consumano numerosi beni, e vogliamo ricorrere all'analogia della bilancia, dobbiamo immaginare che a ciascuno dei due bracci della bilancia 
siano appesi tanti piatti quanti sono i beni (tre nella Figura), come in Figura 4. Le due quantità di ogni bene (ossia la quantità domandata e la quantità offerta) sono poste su due piatti collocati ad uguale distanza dal fulcro della bilancia, ovviamente su bracci diversi; si ha equilibrio economico quando le quantità dei diversi beni sono distribuite in modo tale che la bilancia risulta essere in equilibrio(fisico), con la complicazione che due beni distinti, ma di peso uguale, hanno influenze diverse sulla configurazione di equilibrio (essendo poste a distanze diverse dal fulcro della bilancia economica); ${ }^{5}$ questo fatto complica ulteriormente e notevolmente la ricerca di una opportuna distribuzione dei pesi in grado di porre in equilibrio la bilancia medesima.

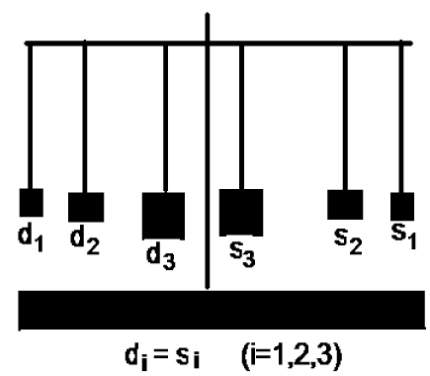

Figura 4. Bilancia economica.

\section{Pareto ingegnere ed ECONOMista}

Vilfredo Pareto, di madre francese e padre italiano, si laureò in ingegneria al Politecnico di Torino nel 1869, con una tesi dal titolo Principi fondamentali della teoria della elasticità dei corpi solidi e ricerche fondamentali sulla integrazione delle equazioni differenziali che ne definiscono l'equilibrio. Questo titolo sottolinea come la nozione (meccanica) di equilibrio fosse tra i primissimi concetti trattati e sviluppati da Pareto ingegnere. Non deve quindi sorprendere se, in modo ricorrente, l'idea di equilibrio abbia pervaso anche la successiva carriera di Pareto economista. E in tema di equilibrio economico, che cosa abbiamo di

5 Appare evidente l'analogia con il cosidetto momento di una forza, rappresentato essenzialmente dal prodotto della intensità della forza per il suo braccio. 
più pertinente della teoria dell'equilibrio (economico) generale? Pertanto i successivi parr. vertono su taluni aspetti di questa teoria.

Il pensiero definitivo di Pareto economista é affidato alla voce Economia matematica, che il nostro scrisse per l'enciclopedia di scienze matematiche, la cui versione originale é in Tedesco, ma venne ben presto ampliata da Pareto e tradotta in Francese, e nel 1955 pure in Inglese.

In questa sede conviene semplicemente sottolineare alcuni punti della voce enciclopedica, dai quali traspare il frequente accostamento che Pareto propone tra teoria economica e meccanica.

A pag. 53 dell'edizione Inglese citata in bibliografia si legge (traduzione in Italiano dello scrivente):

Una posizione degli individui considerati é chiamata posizione di equilibrio se é tale che, secondo le leggi assegnate, gli individui possono continuare indefinitamente in quello stato. Come nella meccanica, vi é l'opportunità di considerare sia equilibri stabili che instabili.

Un secondo esplicito richiamo alla meccanica si trova a pag. 79:

É pertanto del tutto impossibile elencare tutte le interrelazioni dei sistemi economici come lo é l'elencazione di tutti quelli dei sistemi della meccanica.

Infine, si legge a pag. 83:

Le obiezioni che sono state sollevate contro lo studio dell'economia matematica non hanno ne più ne meno valore di quelle precedentemente sollevate contro lo studio della meccanica teorica, ... .

Nel nostro Paese é doveroso ricordare, come seguaci di Pareto, almeno Amoroso (1942) e Vinci $(1944,1945)$. In un volume che raccoglie le lezioni da lui svolte presso l'Istituto Nazionale di Alta Matematica, Amoroso sviluppa connessioni fra meccanica e teoria economica, con particolare enfasi sull'equilibrio generale. In modo specifico egli discute una nozione di potenziale economico e presenta una interpretazione in termini di energia del principio marginalistico; identifica inoltre il principio economico del minimo mezzo con il principio meccanico della minima azione.

Vinci presenta una sua versione dell'equilibrio economico generale sotto un duplice profilo. Egli tratta estesamente sia il caso, più tradizionale, in cui si ipotizza valida la proprietà privata dei mezzi di pro- 
duzione, sia il caso, generalmente trascurato dagli studiosi dell'equilibrio generale, in cui i mezzi di produzione sono proprietà della collettività e lo Stato gestisce l'economia allo scopo di rendere massimo un indice di utilità collettiva.

\section{EQUILIBRIO GENERALE CONCORRENZIALE}

I problemi con i quali si confronta la teoria economica sono sicuramente numerosi; ${ }^{6}$ ma il problema centrale é sempre stato considerato essere questo: come si determinano i prezzi dei beni, e attraverso quali meccanismi. Una risposta rigorosa é offerta dalla teoria dell'equilibrio generale, da decenni considerata il paradigma di riferimento nell'ambito degli studi economici. Questa teoria fu iniziata da Léon Marie Ésprit Walras (1874-77) e proseguita vigorosamente da Pareto, ed é detta scuola di Losanna in quanto entrambi furono professori in quella università.

L'equilibrio generale permette concettualmente di collegare tra loro i mercati di tutti i beni, attraverso le domande e le offerte che scaturiscono dalle decisioni di tutti gli agenti economici (consumatori e imprese) operanti in un dato sistema economico. I prezzi dei beni sono determinati in modo tale che per ciascun bene si verifichi l'uguaglianza fra quantità domandata e quantità offerta. Quando anche per un solo bene si ha diseguaglianza, occorre riaggiustare non solo il prezzo di quel bene, ma anche gli altri prezzi, poiché generalmente le quantità domandate e offerte di ogni bene dipendono anche dai prezzi di altri beni. É questa caratteristica che conferisce la specifica impronta all'equilibrio generale.

Nelle sue linee essenziali, l'equilibrio generale concorrenziale si può rappresentare come segue. Sia $n$ il numero dei beni esistenti, $p_{i}(i$ $=1,2, \ldots, n)$ il prezzo non negativo del bene $i$-mo, $f_{i}\left(p_{1}, p_{2}, \ldots, p_{n}\right)$ la funzione di domanda di questo bene, derivante dai gusti degli individui, e $g_{i}\left(p_{1}, p_{2}, \ldots, p_{n}\right)$ la corrispondente funzione di offerta, dipendente dagli ostacoli incontrati nella produzione. Queste $2 n$ funzioni sono continue, positivamente omogenee di grado zero, e verificano la cosidetta legge di Walras, vale a dire l'uguaglianza $\Sigma_{i} p_{i} f_{i}\left(p_{1}, p_{2}, \ldots, p_{n}\right)=\Sigma_{i} p_{i} g_{i}\left(p_{1}, p_{2}, \ldots, p_{n}\right)$ quali che siano i prezzi. Una configurazione di equilibrio generale é co-

6 Crescita, distribuzione del reddito, fluttuazioni cicliche, scambi internazionali, circolazione monetaria, ... . 
stituita da prezzi non negativi, $p_{i}^{*}$, soddisfacenti, per ogni indice $i$, le condizioni

$$
f_{i}\left(p_{1}^{*}, p_{2}^{*}, \ldots, p_{n}^{*}\right) \leq g_{i}\left(p_{1}^{*}, p_{2}^{*}, \ldots, p_{n}^{*}\right), \quad p_{i}^{*} f_{i}^{*}=p_{i}^{*} g_{i}^{*}
$$

Queste relazioni affermano che in equilibrio per ogni bene la quantità domandata non può eccedere la quantità offerta, ma se la prima é minore della seconda allora il corrispondente prezzo di equilibrio é uguale a zero (regola dei beni cosidetti liberi).

Nell'interpretazione corrente il sistema ora scritto si ritiene rappresentare l'intero sistema economico in un singolo periodo temporale e con certezza; ma nulla vieta di considerare $n$ come numero di beni distinti in base sia alle loro caratteristiche fisiche, chimiche, biologiche, sia alla data in cui ogni bene é reso disponibile per essere consumato oppure impiegato nella produzione, sia all'incertezza dell'ambiente economico scelto dalla Natura ad ogni data fra un numero finito di alternative. ${ }^{7}$ Quindi il precedente sistema matematico può idealmente rappresentare l'intero sistema economico lungo una successione di scadenze temporali, purché l'equilibrio sia completamente determinato sin dal primo periodo. Questa considerazione si può prendere a prestito per introdurre le generalizzazioni sommariamente richiamate nel prossimo paragrafo.

\section{Ampliamenti}

La grande flessibilità concettuale della teoria dell'equilibrio generale é abbondantemente illustrata dai successivi ampliamenti seguiti alla definitiva formalizzazione dell'equilibrio generale concorrenziale a opera di Arrow e Debreu (1954), entrambi studiosi provenienti dalla matematica. Tra i principali ampliamenti conviene sottolineare i seguenti: ${ }^{8}$

1. Equilibrio generale con monopoli e concorrenza monopolistica.

2. Equilibrio generale con prezzi rigidi e meccanismi di razionamento.

3. Equilibrio generale con generazioni sovrapposte.

4. Equilibrio generale dinamico temporaneo e aspettative soggettive.

Si veda Debreu (1959, cap. 7).

Per una presentazione di questi argomenti si può consultare Nicola (2000). 
5. Equilibrio generale approssimato senza convessità nelle preferenze individuali e nelle tecnologie.

6. Equilibrio generale con una infinità di agenti e concorrenza perfetta.

7. Equilibrio generale e incertezza.

8. Mercati incompleti e finanza.

Qualche breve notizia su ognuno di questi ampliamenti può essere opportuna in questa sede.

1. Pareto aveva già introdotto il monopolio nella teoriaWalrasiana dell'equilibrio generale, mentre le prime analisi rigorose sulla concorrenza monopolistica si devono a Negishi (1961). Un'analisi recente, tra le numerose altre, corredata da alcune simulazioni numeriche, é proposta da Nicola(1994).

2. I prezzi rigidi e conseguente razionamento, almeno per quanto riguarda il tasso di salario, erano stati portati all'attenzione degli economisti dal Keynes nella sua opera maggiore del 1936, senza peraltro alcuna sostanziale formalizzazione. Nel modello di equilibrio generale il primo autore ad interessarsene con un'adeguata formalizzazione é stato Glustoff (1968), che ha dimostrato l'esistenza di soluzioni in un modello di tipo Keynesiano dove sono presenti beni i cui prezzi sono rigidi.

3. Il primo modello con generazioni sovrapposte di consumatori, formulato per poter introdurre in modo adeguato una moneta segno nell'equilibrio generale, é frutto del lavoro di Samuelson (1958), preceduto però di alcuni anni da Allais(1947). Uno studio attuale di questi modelli é proposto da Aliprantis, Brown e Burkinshaw (1989, cap .5).

4. L'equilibrio generale temporaneo era stato anticipato in nuce dallo studioso svedese Lindahl(1929), e ripreso vigorosamente e diffuso da Hicks $(1939,1946)$, per sostituire la generale mancanza di mercati per i beni futuri con funzioni di aspettative soggettive, per mezzo delle quali gli agenti economici si formano delle previsioni su quali potranno essere i prezzi futuri, previsioni necessarie per scegliere programmi ottimi intertemporali. Una significativa raccolta di saggi sull'equilibrio temporaneo é Grandmont(1988).

5. L'equilibrio generale approssimato, definito in assenza di ipotesi di convessità sulle funzioni di utilità e sugli insiemi di tecnologie, determina un vettore di prezzi in corrispondenza del quale quasi tutti (ma non tutti) gli agenti ottengono il massimo risul- 
tato. La proprietà matematica su cui si basa la dimostrazione di esistenza di soluzioni si fonda sul risultato, ottenuto da Shapley e Folkman, ${ }^{9}$ che nello spazio euclideo $n$-dimensionale la somma (algebrica) di numerosi (rispetto ad $n$ ) insiemi é praticamente un insieme convesso. Essenzialmente, questo significa che al più sono $n$ gli agenti economici che ottengono un vettore di beni diverso da quello che rende massime le loro funzioni obiettivo; quindi, se il numero degli agenti é molto più grande di $n$, quasi tutti costoro ottengono quanto hanno liberamente scelto, mentre sono pochi gli agenti che ottengono un risulato diverso da quello che rende massima la loro funzione obiettivo (utilità $o$ profitto).

6. La principale forma di mercato su cui si basa la teoria dell'equilibrio generale é la concorrenza perfetta. In termini rigorosi dal punto di vista matematico questa forma di mercato richiede che per ogni bene vi sia un numero infinito di agenti, ciascuno di peso nullo (nel senso della teoria della misura di Lebesgue) ma complessivamente di peso positivo. Il primo economista ad occuparsene in modo rigoroso é stato Aumann(1964).

7. L'incertezza rappresenta una componente ineliminabile della vita economica quando si considerano decisioni che riguardano il futuro, sia di un'intera economia quanto di ogni agente economico. Nella teoria dell'equilibrio generale un passo interessante é stato compiuto da Dréze (1985), che ha introdotto l'incertezza nell'analisi del comportamento dell'impresa, dove l'importanza di una programmazione intertemporale é assolutamente indispensabile.

8. Nelle economie reali per la maggioranza dei beni non esistono mercati futuri attivi nel periodo corrente. Di questa mancanza tiene conto l'equilibrio generale temporaneo di cui si é detto sopra. Un altro modo di affrontare la questione é introdurre nei modelli di equilibrio generale un certo numero di mercati futuri posti in essere attraverso l'introduzione di beni artificiali, le cosidette attività finanziarie, che in parte servono a coprire l'incertezza sui prezzi futuri. Un buon riferimento allo studio dell'equilibrio con mercati incompleti é il volume Magill e Quinzii (1996).

9 Per una dimostrazione si veda Starr (1969). 


\section{EQUILIBRIO GENERALE APPLICATO}

É bene ricordare che i dati, su cui sono basate le applicazioni dei modelli economici, sono sempre rilevati in modo approssimativo. Inoltre in economia, a differenza di quanto generalmente accade in altre discipline quali la fisica, sono quasi del tutto impossibili gli esperimenti, sia sul campo che in laboratorio. Questo rende ulteriormente complicato lo studio dei fenomeni economici e la formulazione di previsioni attendibili.

Ciò detto, nonostante la sua indubbia complessità formale, a partire dal Leontief (1936) si sono susseguite le applicazioni di modelli di equilibrio generale a numerose economie del mondo concreto. Per Leontief lo scopo principale della sua analisi delle interdipendenze strutturali é di riuscire a semplificare drasticamente il modello di equilibrio generale walrasiano fino a renderlo concretamente applicabile.

Per dimostrare l'esistenza di un equilibrio generale si impiega di solito un teorema del punto fisso, per esempio il teorema di Kakutani; ma per applicare l'equilibrio generale a casi concreti é necessario munire i teoremi del punto fisso di algoritmi per il loro calcolo. A questo compito ha provveduto lo Scarf(1973), ${ }^{10}$ che presenta anche qualche applicazione numerica, peraltro non tratta da alcuna economia concreta.

Un buon contributo sulle applicazioni dell'equilibrio generale ad economie del nostro mondo é costituito dagli esempi proposti nella Parte II del volume di Shoven e Whalley (1992) dove si progetta un modello di equilibrio generale applicabile e lo si applica agli Stati Uniti d'America. Anche Morishima (1972) e collaboratori, per fare un altro esempio, hanno proposto applicazioni dell'equilibrio generale a casi concreti, tra cui gli Stati Uniti d'America e il Giappone. Si segnala inoltre il volume curato da Scarf e Shoven (1984) che contiene, fra l'altro, un'applicazione dell'equilibrio generale all'economia messicana e un'altra applicazione all'economia australiana. Il volume contiene inoltre due saggi che studiano, il primo il problema della specificazione numerica e il secondo i metodi econometrici per l'analisi dei modelli di equilibrio generale applicato.

$10 \mathrm{Ma}$, almeno concettualmente, il problema di calcolo delle soluzioni era stato messo a fuoco, molto tempo prima, da Barone (1908). 


\section{COMPETIZIONE: DALLA MECCANICA ALLA BIOLOGIA}

Conviene ora soffermarsi brevemente sul termine concorrenza, in Inglese "competition", ossia competizione. Anche se la teoria dell'equilibrio generale non ne fa menzione esplicita, la nozione di competizione richiama le idee di Darwin sulla selezione ed evoluzione delle specie, e quindi fa riferimento alla biologia.

I prezzi di equilibrio emergono, in definitiva, dal conflitto di interessi, prima di tutto fra imprese e consumatori (le specie), ma poi anche all'interno della sfera delle imprese e di quella dei consumatori (gli individui). Dunque, anche se la nozione di equilibrio generale é indubbiamente presa a prestito dalla fisica, nel suo intimo vi é un fondamento biologico indiscutibile e ineliminabile. Una trattazione interessante sulle teorie evolutive in economia é oggetto del volume di Nelson eWinter(1981).

\section{PREVISIONI}

In queste note si é praticamente discusso solo di equilibrio generale; quale potere previsivo può avere? Innanzitutto il quesito deve riferirsi ai modelli di equilibrio generale applicato ricordati poco sopra. Il loro potere previsivo potrebbe essere buono se la struttura dell'economia permanesse davvero immutata per un certo numero di anni; ma questo accade difficilmente. Dunque non é nell'equilibrio generale che si può fare affidamento a fini previsivi. Per questo scopo sono molto più adatti i modelli macroeconomici dinamici che, proprio per il fatto di rappresentare in forma estremamente aggregata un'economia, meglio si prestano a prevedere quantitativamente futuri andamenti delle principali grandezze economiche aggregate, quali i consumi, gli investimenti, il tasso di crescita, ... .

\section{CONSIDERAZIONI FINALI}

Sembra di poter concludere le precedenti note affermando che i fondamenti della meccanica, assai più delle analogie di carattere biologico, sono stati la principale fonte di ispirazione per i cultori della teo- 
ria economica, ${ }^{11}$ e presumibilmente continueranno a esserlo. Questo non significa escludere a priori la possibilità di ricorrere ad analogie biologiche quando queste possono contribuire ad illuminare il divenire di un sistema economico.

In particolare, con riferimento al problema delle previsioni si é sostenuto che i modelli di equilibrio generale mal si prestano allo scopo; invece i modelli macrodinamici, per la loro relativa semplicità e buona flessibilità, si rivelano assai più adatti. Forse si potrebbe pensare di costruire e stimare modelli uniperiodali di equilibrio generale, sintetizzarli con corrispondenti modelli macrostatici e da questi passare a corrispondenti modelli macrodinamici; impiegare poi questi ultimi a scopi previsivi e risalire dai loro risultati a possibili modelli di equilibrio generale multiperiodale che li hanno generati, per ottenere da questi ultimi previsioni più articolate. Ma un programma di ricerca come questo per il momento sembra essere del tutto prematuro.

11 Almeno di quella attualmente predominante. 


\section{BIBLIOGRAFIA}

Aliprantis, C. D., D. J. Brown e O. Burkinshaw (1989), Existence and Optimality of Competitive Equilibria, Berlino e Heidelberg, Springer-Verlag.

Allais, M. (1947), Économie et Intérêt, Parigi, Imprimerie Nationale.

Amoroso, L.(1942), Meccanica economica, Città di Castello, Macrí.

Arrow, K.J. e G. Debreu (1954), Existence of an Equilibrium for a Competitive Economy, Econometrica, vol. 22, pp. 265-290.

Aumann, R. J. (1964), Markets with a Continuum of Traders, Econometrica, vol. 32, pp.39-50.

Barone, E. (1908), Il ministro della produzione nello stato collettivista, Giornale degli Economisti, vol. 37, pp. 267-293, 391-414.

Cournot, A. (1838), Recherches sur les principes mathématiques de la théorie des richesses, Parigi, Hachette.

Debreu, G. (1959), Theory of Value: An Axiomatic Analysis of Economic Equilibrium, New York, Wiley.

Dréze, J.H.(1985), Uncertainty and the Firm in General Equilibrium, Economic Journal, vol. 95 (supplemento), pp. 1-20.

Glustoff, E. (1968), On the Existence of a Keynesian Equilibrium, The Review of Economic Studies, vol. 35, pp. 327-334.

Grandmont, J.M. (1988) (a cura di), Temporary Equilibrium. Selected Readings, San Diego (CA), Academic Press.

Hicks, J.R. (1939) Value and Capital, Oxford, at the Clarendon Press (seconda edizione 1946).

Leontief, W.W. (1936), Quantitative Input and Output Relations in the Economic System of the United States, Review of Economic Statistics, vol. 60, pp. 105-125.

Magill, M.J.P. e M. Quinzii (1996), Theory of Incomplete Markets, Cambridge, The MIT Press.

Marshall, A. (1920), Principles of Economics. An Introductory Volume, Londra, Macmillan (prima edizione 1890).

Modigliani, F. e R. Brumberg (1954), Utility Analysis and the Consumption Function: an Interpretation of Cross-Section Data, in K.K. Kurihara (a cura di), Post Keynesian Economics, pp. 388-436.

Morishima, M., Y. Murata, T. Nosse e M. Saito (1972), The Working of Econometric Models, Cambridge, Cambridge University Press. 
Negishi, T. (1961), Monopolistic Competition and General Equilibrium, The Review of Economic Studies, vol. 28, pp. 195-201.

Nelson, R. e S. Winter (1981), An Evolutionary Theory of Economic Change, Cambridge (MA), Harvard University Press.

Nicola, P.C. (1994), Imperfect General Equilibrium, Lecture Notes in Economics andMathematical Systems 415, Berlino, Springer-Verlag.

Nicola, P.C.(2000), Mainstream Mathematical Economics in the $20^{\text {th }}$ Century, Berlino, Springer, capp. 1, 2, 11, 16-19, 26.

Pareto, V. (1906, 1909), Manuale di economia politica, edizione critica a cura di A. Montesano, A. Zanni e L. Bruni, Milano, Università Bocconi Editori, 2006.

Pareto,V. (1911), Ėconomie mathématique, Encyclopédie des Sciences Mathématiques, tomo I, vol. IV, fasc. 4, Parigi, Gauthier-Villars, pp.591-640 (voce ampliata rispetto all'originale in Tedesco).

Penrose, E.T. (1952), Biological Analogies in the Theory of the Firm, American Economic Review, vol. 42, pp. 804-819.

Samuelson, P.A. (1958), An Exact Consumption-Loan Model of Interest with or without the Social Contrivance of Money, Journal of Political Economy, vol. 66, pp. 467-482.

Scarf, H.E. (1973), The Computation of Economic Equilibria, New Haven, Yale University Press.

Scarf, H.E. e J.B. Shoven (1984), Applied General Equilibrium Analysis, Cambridge, Cambridge University Press.

Shoven, J.B. e J. Whalley (1992), Applying General Equilibrium, Cambridge, Cambridge University Press.

Simon, H.A. (1955), A Behavioural Model of Rational Choice, Quarterly Journal of Economics, vol. 69, pp. 99-118.

Smith, A. (1776) The Wealth of Nations, Methuen, University Paperbacks, 1961.

Starr, R.M.(1969), Quasi-Equilibria in Markets with non Convex Preferences, Econometrica, vol. 37, pp. 25-38.

Vinci, F. (1944-45), Gli ordinamenti economici, Milano, Giuffré (due voll.).

Walras, L.(1874-77), Éléments d'économie politique pure ou théorie de la richesse sociale, Parigi, Pichon et Durand-Auzias, 1900. 